\title{
Trends des Tabak- und Alkoholkonsums über 65 Jahre in Deutschland
}

\section{Trends of Tobacco and Alcohol Consumption over 65 Years in Germany}

(c) (1) $(9$

\section{Autoren}

Ulrich John', 2, Monika Hanke ${ }^{1}$

Institut

1 Institut für Sozialmedizin und Prävention, Universitätsmedizin Greifswald

2 Deutsches Zentrum für Herz-Kreislauf-Forschung (DZHK)

Schlüsselwörter

Tabakkonsum, Alkoholkonsum, Trends, Prävention, Gesetze

Key words

tobacco consumption, alcohol consumption, trends, prevention, laws

\section{Bibliografie}

DOI https://doi.org/10.1055/s-0043-110854

Online-Publikation: 26.7.2017

Gesundheitswesen 2018; 80: 160-171

(c) Georg Thieme Verlag KG Stuttgart · New York

ISSN 0941-3790

\section{Korrespondenzadresse}

Prof. Ulrich John

Institut für Sozialmedizin und Prävention

Universitätsmedizin Greifswald

Walther-Rathenau-Straße 48

17475 Greifswald

ujohn@uni-greifswald.de

\section{ZUSAMMENFASSUNG}

Ziel Für Deutschland lagen keine Tabak- und Alkoholverbrauchsschätzungen aufgrund von Vermarktungsdaten vor, die öffentlich und für Analysen zeitlicher Trends geeignet sind. Entwicklungen der Tabak- und Alkoholkonsummengen in Deutschland für die Jahre 1950-2014 sollten geschätzt werden.

Methodik Daten über den Tabak- und Alkoholverbrauch der Bundesrepublik Deutschland pro Kalenderjahr von 1950 bis 2014 zu Reinalkoholmengen wurden aufgrund von Meldungen der Produzenten von Bier, Wein und Spirituosen an Steuerbehörden und das Statistische Bundesamt gewonnen. Zeitliche Trends über die 65 Kalenderjahre wurden mithilfe von Joinpoint-Analysen ermittelt.
Ergebnisse Der Tabakkonsum stieg von 1950 bis 1972 an und sank danach, überwiegend mit 1,2-6,9 Prozentpunkten pro Jahr, bis zum Ende des Beobachtungszeitraumes 2014. Der Alkoholkonsum stieg bis zum Jahr 1974 an und sank danach mit 1,0 Prozentpunkten jährlich bis zum Ende des Beobachtungszeitraumes 2014.

Schlussfolgerungen Erklären lassen sich die Befunde u.a. mit Änderungen sozialer Normen zum Konsum nach Gesetzänderungen, zu Tabakrauchen u. a. durch Steuererhöhungen, Nichtraucher- und Jugendschutz, zu Alkoholkonsum u. a. durch Gesetze der Sicherheit im Straßenverkehr. Nach Einführung einzelner gesetzlicher Leistungen war eine Beschleunigung der Senkung des Tabakrauchens beobachtbar. Die Tabak- und Alkoholkonsummengen in Deutschland sind dennoch im internationalen Vergleich nach wie vor hoch am Ende des Beobachtungszeitraumes 2014.

\section{ABSTRACT}

Aim No estimation was available for tobacco and for alcohol consumption in Germany based on sales data that were provided for public use and suited for time trend analysis.

Objectives To estimate trends of tobacco and alcohol consumption rates for the years 1950-2014.

Methods Data on tobacco and alcohol consumption in the nation were retrieved from reports made by producers of beer, wine, or spirits to the Federal Statistics Office of Germany. Time trends over the 65 years were calculated using the program Joinpoint.

Results Tobacco consumption rose from 1950 to 1972 . Thereafter it decreased, mostly by 1.2-6.9 percentage points per year. Alcohol consumption rose until the year 1974 and decreased thereafter by 1.0 percentage points annually until the end of the time period under analysis in 2014.

Conclusions The findings may be explained, among others, by changes of social norms according to smoking and alcohol consumption after tax increases, nonsmoker and youth protection laws, and legislative measures against driving under the influence of alcohol. A steepening of the decrease in tobacco consumption occurred after laws including tax increases had come into effect. However, the tobacco and alcohol consumption levels were still high at the end of the observation period in 2014. 


\section{Einleitung}

Zeitliche Trends von Tabak- und Alkoholkonsummengen in Nationen gehören zu Outcome-Parametern von Prävention [1]. Sie lassen sich durch Daten der Vermarktung (Steuer- und Herstellerdaten) schätzen, die zeigen, wie viel Tabakwaren und wie viel Alkohol in welcher Art von Getränk den Verbrauchern zur Verfügung gestellt werden. Steuerdaten sind nutzbar bei Tabakwaren, Bier, Schaumwein, Zwischenerzeugnissen, wie Sherry oder Portwein, sowie bei Spirituosen. Bei Wein handelt es sich um Meldungen der Hersteller. Wein unterliegt in Deutschland keiner Besteuerung. Vermarktungsdaten können der Schätzung der Tabak- und Alkoholmengen dienen, die von den Verbrauchern in Deutschland erworben werden. Relationen nationalen Tabak- und Alkoholkonsums mit weiteren Merkmalen, insbesondere Morbidität und Mortalität, aber auch potenziellen sozialen und wirtschaftlichen Folgen des Tabak- und Alkoholkonsums lassen sich berechnen. So zeigen Befunde Zusammenhänge zwischen dem Gesamtalkoholkonsum einer Nation einerseits und exzessivem Konsum sowie alkoholbezogenen Schäden andererseits [2].

Vermarktungsdaten bilden neben Personenbefragungen eine Informationsquelle über den Tabak- und Alkoholkonsum einer Nation. Sie erlauben aber keine Aussagen über Subgruppen der Bevölkerung, z. B. nach Geschlecht oder Lebensalter. Vermarktungsdaten weisen andere Verzerrungen der Aussagen auf als Daten von Bevölkerungsbefragungen. Vermarktungsdaten lassen zollfreie Einfuhr, Schmuggel, Verschenken von Alkohol in das Ausland sowie nicht konsumierten Alkohol unberücksichtigt [vgl. [3]. Das Ausmaß dieser Verzerrungen der Schätzung des Alkoholkonsums in der Bevölkerung ist nicht bekannt. Sie sind als geringfügig zu erwarten in Nationen, die bezogen auf das Einkommen niedrige Preise für Alkohol haben. Das trifft für Deutschland zu [4]. Dem stehen Verzerrungen von Surveydaten gegenüber, v. a. Fehlberichte der Befragten durch Nennung geringeren Konsums als tatsächlich erfolgt (underreporting) [5]. Das Ausmaß lässt sich schätzen durch einen systematischen Vergleich von Ergebnissen staatlicher Statistiken aufgrund von Vermarktungsdaten und Ergebnissen aus Bevölkerungssurveys. Deutschland und Frankreich hatten den höchsten Konsum aufgrund der Statistiken, aufgrund eines Surveys den niedrigsten. Er betrug in Deutschland 31\% und in Frankreich 36\% des Konsums, der aufgrund der Vermarktungsdaten in offiziellen Statistiken zu erwarten war [2].

Tabak- und Alkoholkonsum sind miteinander verbunden [6, 7]. Sie gemeinsam zu analysieren ist aus Gründen sinnvoll, die durch epidemiologische, klinische und psychologische Evidenz dargelegt wurden [6]. Z. B. zeigte epidemiologische Forschung, dass Menschen mit problematischem Alkoholkonsum wahrscheinlicher auch Raucher sind als Menschen ohne problematischen Alkoholkonsum. Mit der Zahl der Zigaretten steigt die Wahrscheinlichkeit einer Alkoholabhängigkeit [6]. Klinische Evidenz belegt, dass Menschen mit gesundheitlichen Schäden durch Alkohol auch überdurchschnittlich wahrscheinlich Tabakraucher sind. Unter Patienten, die wegen Alkoholproblemen behandelt wurden, waren mehr als $75 \%$ auch Tabakraucher [8]. Psychologische Evidenz zeigt, dass Alkoholkonsum das Bedürfnis Tabak zu rauchen steigert (vgl. (6]).

Deutschland weist im internationalen Vergleich hohen Tabakund hohen Alkoholkonsum auf. Für das Jahr 2014 wurde die Zahl der Zigaretten pro Bundesbürgerin oder -bürger im Alter ab 15 Jahren mit 1480 angegeben, höher als in präventionsaktiveren Ländern, wie Frankreich (993 Zigaretten), Schweden (831 Zigaretten), England (827 Zigaretten), Norwegen (556 Zigaretten) und Island (551 Zigaretten) [9]. Der Alkoholkonsum Deutschlands entspricht dem neunten Rang unter 40 Mitglieds- oder Partnerländern der Organisation für wirtschaftliche Zusammenarbeit und Entwicklung (OECD) [10].

Limitationen des bisherigen Wissens bestehen darin, dass für Deutschland keine Tabak- und Alkoholverbrauchsschätzungen aufgrund von Vermarktungsdaten vorlagen, die 3 Kriterien erfüllen: Nachprüfbarkeit, je ein Mengenwert für den Tabak- und den Alkoholkonsum der Nation pro Kalenderjahr sowie Eignung für Analysen zeitlicher Trends. Die Nachprüfbarkeit bisheriger Daten zum Alkoholkonsum ist ungenügend.

Als Schätzer des gesamten Tabak- und Alkoholkonsums der Bundesrepublik Deutschland wurde seit Jahren je ein Mengenwert veröffentlicht $[11,12]$. Zum Tabakkonsum wurde aber keine einheitliche Umrechnung von losem Rauchtabak in Zigarettenäquivalente vorgenommen. Tabak- und Alkoholkonsum wurden pro Bundesbürgerin oder -bürger angegeben, nicht standardisiert für die Bevölkerung im Lebensalter, in dem überwiegend Tabak und Alkohol konsumiert wird, im Lebensalter ab 15 Jahren. Dieses Alter ist international üblich bei Darstellungen des Alkoholkonsums. Es erleichtert Vergleiche mit anderen Nationen [10,13]. Die bisher publizierten Mengenwerte zu Alkohol basieren zu wenig auf verfügbarer Evidenz. Für Bier wurden international überwiegend 4-5 Volumen-\% Alkohol, für Wein 11-16 Volumen-\% Alkohol und für Spirituosen 40 Volumen-\% Alkohol angenommen [10,13]. Durch die Spannweite dieser Grenzen und durch die Marktvielfalt sind diese Schätzungen unzureichend. Für Deutschland liegt seit dem Jahr 2000 die Empfehlung einer Arbeitsgruppe von Wissenschaftlern und Vertretern der Bier und der Spirituosen herstellenden Industrie vor, pauschal Bier mit 4,8, Wein oder Sekt mit 11,0 und Spirituosen mit 33,0 Volumen-\% Alkohol zu werten [14]. Diese Prozent-Angaben entstanden durch Einigungen. Eine empirische Grundlage ist öffentlich nicht zugänglich (vgl. [11]). Die Berechnung zeitlicher Trends erfordert, dass für alle Kalenderjahre die gleichen Kriterien für die Schätzung galten.

Ziel der Studie war, zeitliche Trends der Tabak- und Alkoholkonsummengen in Deutschland für die Jahre 1950-2014 zu schätzen.

\section{Methoden}

\section{Daten}

Die verwendeten Daten des Tabak- und des Alkoholkonsums stammen von Erzeugern. Die Daten sind Veröffentlichungen des Statistischen Bundesamtes entnommen. Sie enthalten die Angaben der Erzeuger über die Hauptzollämter oder im Fall von Wein über die Statistischen Landesämter, denen die Weinerzeugermeldungen vorliegen. Zum Tabakrauchen nutzten wir Mengengaben mit der Zahl versteuerter Zigaretten, Zigarren, Zigarillos sowie Tonnen Feinschnitt- und Pfeifentabak [15-17]. Die Tabakmengen rechneten wir in Zigarettenäquivalente um auf der Grundlage einer Empfehlung der OECD. Danach werden eine Zigarette, ein Gramm Feinschnitt- oder ein Gramm Pfeifentabak als ein Zigarettenäquivalent und Zigarren oder Zigarillos als 2 Zigarettenäquivalente gewertet [18]. Zu Kautabak fanden wir keine verwertbaren Daten. 
Für Bier nutzten wir die jährlich in den Handel gebrachen Mengen aufgrund von Meldungen der Hauptzollämter an das Statistische Bundesamt [16, 19-55]. Die Menge an produziertem Weinmost und Wein wird aus der Meldung der Erzeuger an die Statistischen Landesämter aufgrund der Traubenernte- und Weinerzeugungsmeldung gemäß EU- und Bundesrecht weiter an das Statistische Bundesamt übermittelt [16, 17, 55-85]. Für die Bestimmung des Alkoholgehaltes verwendeten wir die jährlichen Mostgewichte von Rot- und Weißwein [59-85]. Die Meldung der Weinerzeuger erfolgt in Wirtschaftsjahren, die nicht mit Kalenderjahren übereinstimmen. Angaben zu Schaumwein und Zwischenerzeugnissen werden über Steuerdaten aus den Hauptzollämtern vom Statistischen Bundesamt zusammengefasst mit den Meldungen über die produzierten Weinmengen zur Verfügung gestellt. Wein, der für Branntwein oder nicht zu Trinkzwecken produziert wurde, blieb bei Alkohol aus Wein unberücksichtigt. Für die Schätzung des Reinalkohols aus Spirituosen nutzten wir Angaben des Statistischen Bundesamtes $[16,17,55]$. Für Spirituosen wird im Rahmen der Branntweinsteuerstatistik die Menge verarbeiteten reinen Alkohols über die Hauptzollämter an das Statistische Bundesamt gemeldet. Export und Import von Bier, Wein, Schaumwein, Zwischenerzeugnissen sowie Spirituosen sind in den Angaben des Statistischen Bundesamtes und in unseren Schätzungen berücksichtigt.

\section{Alkoholmengen}

Die in Deutschland pro Kalenderjahr konsumierten Mengen Reinalkohols wurden zunächst für Bier, Wein und Spirituosen getrennt geschätzt. Für Bier lagen Angaben zu Hektolitern vor, seit dem Jahr 1956 differenziert nach Stammwürze. Aus der Stammwürze schätzten wir Volumen-Prozent und Liter Reinalkohol [86]. Die Stammwürze ist in Grad Plato mit variierender Genauigkeit angegeben, für die Jahre 1956-1992 in 2-5,5 (Einfachbier), 7-8 (Schankbier), 11-14 (Vollbier), 16 und mehr (Starkbier). Für die Jahre 1986-1992 waren nur Angaben als ober- und untergäriges Einfach-, Voll- und Starkbier verfügbar, für 1993-1997 aufgrund des Biersteuergesetzes von 1993 jeweils folgende Grade Plato: 1-6, 7, 8, 9, 10, 11, 12, 13, 14 oder höher. Für 1998-2014 lagen die Angaben weiter differenziert vor $(1-4,5,6,7,8,9,10,11,12,13,14,15,16,17,18,19$, 20, 21, 22-35 Grad Plato). Die Daten seit 1993 lassen sich als hinreichend genau werten. Aus ihnen wurden die Mengen an Reinalkohol bestimmt [19-28, 30, 32, 34, 36, 38, 40, 42, 44, 46, 48, 50, 52]. Um zu einer Schätzung der Volumen-\% Alkohol für die Jahre 1950 bis 1992 zu kommen, diente als Grundlage, dass in den Jahren 1993-2014 mehr als $90 \%$ des Bieres 11 Grad Plato aufwiesen. In den Jahren 1956-1985 hatten mehr als 98\% des Bieres 11-14 Grad Plato. Aufgrund dieser Informationen werteten wir für die Jahre 1950-1992 die Volumen- \% für Bier pauschal mit 4,8\%.

Ex- und Importe von Bier sind in den Daten des Statistischen Bundesamtes in den Jahren 1950-1992 bereits berücksichtigt. Für die Jahre 1993-2014 berechneten wir differenziert nach Grad-Plato-Gruppen die in Deutschland vermarkteten Biermengen abzüglich der Exporte in EU- und Drittländer und zuzüglich der Importe aus Drittländern [29, 31, 33, 35, 37, 39, 41, 43, 45, 47, 49, 51, 53, 54]. Für diese Teilmengen wurden die gleichen Reinalkoholanteile wie für die in Deutschland vermarktete Biermenge zugrunde gelegt.
Importe aus EU-Ländern sind in den Grad-Plato-Angaben des Statistischen Bundesamtes enthalten.

Biermischungen werden aufgrund des Biersteuergesetzes von 1993 von Bier getrennt erfasst, die Mengen vom Statistischen Bundesamt seit dem Jahr 1997 veröffentlicht [23-28, 30, 32, 34, $36,38,40,42,44,46,48,50,52]$. Biermischungen schätzten wir aufgrund von Angaben der 17 Produzenten, die im Jahr 2014 mehr als eine Million Hektoliter Bier pro Region in Deutschland verkauften [87] mit 2,5 Volumen-\% Alkohol.

Für Wein wurde der Gehalt an Reinalkohol in 3 Schritten ermittelt. Im ersten Schritt bestimmten wir für jedes Kalenderjahr den durchschnittlichen Alkoholgehalt des Weines. Dazu dienten aus der Statistik über den Anbau von Rot- und Weißwein in Deutschland die Öchsle-Grade des Rot- und des Weißmostes. Für die Jahre 1951-2010 (außer 1954) wurden die einschlägigen Statistischen Jahrbücher für die Bundesrepublik Deutschland verwendet [5982]. Für die Jahre 1950 und 1954 fanden wir keine deutschlandweiten Daten und nutzten ausschließlich Daten der Weinmosternte Rheinland-Pfalz [85]. Für 2011-2014 bildeten Daten einer Fachserie des Statistischen Bundesamtes die Grundlage [83, 84]. ÖchsleGrade wurden für die Schätzung der Volumen-\% Reinalkohol im Wein für jedes einzelne Kalenderjahr der Deutschen Weinordnung gemäß [88] zu Grunde gelegt. Im zweiten Schritt wurde der Mengenanteil Alkohol aus Rot- und Weißwein bestimmt, ebenfalls aufgrund des Mostes. Daraus schätzten wir jeweils den Anteil Alkohol aus Rot- und aus Weißwein. Im dritten Schritt wurde nach diesen beiden Anteilen gewichtet die Alkoholmenge von Wein insgesamt pro Kalenderjahr bestimmt. Für die Berechnungen nutzten wir Stata 14.2 [89].

\section{Datenanalyse}

Analysiert wurden die vermarkteten Tabak- und Alkoholmengen und die Bevölkerungszahlen der Bundesrepublik Deutschland für die 65 Jahre von 1950 bis 2014 [90-93]. Für das Jahr 1990, in dem die ehemalige Deutsche Demokratische Republik und die Bundesrepublik Deutschland zu einer Nation vereinigt wurden, sind vom Statistischen Bundesamt keine Daten bereitgestellt. Daher wurde für die Tabak- und Alkoholmengen der Mittelwert der Jahre 1988 und 1989 genutzt. Für die Bevölkerungszahlen legten wir für 1990 die der alten Bundesländer zugrunde. Für Spirituosen im Jahr 2010 setzten wir den Mittelwert der Jahre 2009 und 2011 ein, weil wegen steuerrechtlicher Änderungen Angaben fehlten, die jedoch keinen Einfluss auf unsere Mengenangaben zu Reinalkohol haben. Sowohl Tabak- als auch Alkoholkonsum sind pro Bundesbürgerin oder -bürger im Alter ab 15 Jahren dargestellt ( $\triangleright$ Tab. 1). Der Berechnung zeitlicher Trends diente das Programm Joinpoint, Version 4.1.1 $[94,95]$. Es ermittelt auf regressionsanalytischem Wege Trends, die als signifikant steigend, signifikant sinkend oder bei Nichtsignifikanz als stagnierend beschreibbar sind. Die Stärke der Steigung oder Senkung wird in jährlichen Prozentpunkten beschrieben $[94,95]$. Wir legten maximal 5 Trends fest unter dem Aspekt hinreichender Detailliertheit bei gleichzeitiger Übersichtlichkeit von Trends. Zusätzlich werden Ergebnisse der Joinpoint-Analysen mit maximal 4 Trends dargestellt. 
- Tab. 1 Konsum von Zigarettenäquivalenten und Litern Reinalkohol pro Bundesbürger/in im Alter ab 15 Jahren in den Jahren 1950 bis 2014.

\begin{tabular}{|c|c|c|c|c|c|}
\hline \multirow{2}{*}{ Jahr } & \multirow{2}{*}{$\begin{array}{l}\text { Zigaretten- } \\
\text { äquivalente }^{a}\end{array}$} & \multicolumn{4}{|c|}{ Liter Reinalkohol } \\
\hline & & Bier & Wein & Spirituosen & Gesamt \\
\hline 1950 & 1380 & 2,11 & 0,50 & 1,38 & 3,99 \\
\hline 1951 & 1504 & 2,73 & 0,71 & 1,36 & 4,80 \\
\hline 1952 & 1509 & 3,08 & 1,00 & 1,31 & 5,40 \\
\hline 1953 & 1553 & 3,38 & 1,15 & 1,36 & 5,88 \\
\hline 1954 & 1577 & 3,48 & 0,81 & 1,43 & 5,72 \\
\hline 1955 & 1661 & 3,88 & 0,96 & 1,60 & 6,44 \\
\hline 1956 & 1741 & 4,23 & 0,88 & 1,83 & 6,94 \\
\hline 1957 & 1822 & 4,84 & 0,88 & 1,98 & 7,70 \\
\hline 1958 & 1874 & 5,05 & 0,84 & 2,11 & 8,00 \\
\hline 1959 & 1953 & 5,43 & 1,56 & 2,13 & 9,13 \\
\hline 1960 & 2053 & 5,76 & 1,20 & 2,43 & 9,39 \\
\hline 1961 & 2174 & 6,20 & 1,60 & 2,69 & 10,49 \\
\hline 1962 & 2253 & 6,57 & 1,57 & 3,04 & 11,18 \\
\hline 1963 & 2272 & 6,98 & 1,51 & 3,26 & 11,75 \\
\hline 1964 & 2380 & 7,54 & 1,60 & 3,08 & 12,21 \\
\hline 1965 & 2455 & 7,54 & 1,45 & 3,52 & 12,51 \\
\hline 1966 & 2541 & 7,81 & 2,02 & 3,04 & 12,87 \\
\hline 1967 & 2497 & 7,94 & 1,92 & 2,90 & 12,76 \\
\hline 1968 & 2609 & 8,06 & 1,54 & 3,36 & 12,96 \\
\hline 1969 & 2703 & 8,44 & 1,87 & 3,56 & 13,87 \\
\hline 1970 & 2810 & 8,76 & 1,75 & 3,89 & 14,41 \\
\hline 1971 & 2919 & 8,96 & 2,61 & 4,29 & 15,86 \\
\hline 1972 & 2905 & 8,99 & 2,00 & 3,78 & 14,77 \\
\hline 1973 & 2862 & 9,03 & 2,52 & 3,87 & 15,42 \\
\hline 1974 & 2899 & 9,02 & 2,25 & 3,38 & 14,65 \\
\hline 1975 & 2815 & 9,04 & 2,94 & 3,87 & 15,84 \\
\hline 1976 & 2916 & 9,14 & 3,36 & 4,20 & 16,70 \\
\hline 1977 & 2715 & 8,92 & 2,56 & 3,65 & 15,13 \\
\hline 1978 & 2820 & 8,65 & 2,74 & 3,71 & 15,11 \\
\hline 1979 & 2825 & 8,53 & 2,97 & 4,12 & 15,63 \\
\hline 1980 & 2863 & 8,50 & 2,85 & 3,71 & 15,06 \\
\hline 1981 & 2875 & 8,51 & 2,89 & 3,37 & 14,77 \\
\hline 1982 & 2691 & 8,53 & 2,70 & 3,04 & 14,27 \\
\hline 1983 & 2745 & 8,50 & 3,09 & 2,90 & 14,50 \\
\hline 1984 & 2713 & 8,22 & 2,46 & 2,75 & 13,42 \\
\hline 1985 & 2711 & 8,21 & 3,02 & 2,78 & 14,01 \\
\hline 1986 & 2646 & 8,23 & 2,44 & 2,68 & 13,35 \\
\hline 1987 & 2644 & 8,07 & 2,69 & 2,61 & 13,37 \\
\hline 1988 & 2597 & 8,02 & 3,06 & 2,47 & 13,55 \\
\hline 1989 & 2601 & 7,97 & 3,01 & 2,33 & 13,31 \\
\hline 1990 & 2599b & $7,99^{b}$ & $3,04^{b}$ & $2,40^{b}$ & $13,43^{b}$ \\
\hline 1991 & 2466 & 8,07 & 2,50 & 2,82 & 13,39 \\
\hline 1992 & 2281 & 8,19 & 2,69 & 2,57 & 13,45 \\
\hline 1993 & 2193 & 7,25 & 2,91 & 2,94 & 13,10 \\
\hline 1994 & 2252 & 7,37 & 2,60 & 2,82 & 12,80 \\
\hline 1995 & 2244 & 7,26 & 2,51 & 2,82 & 12,59 \\
\hline 1996 & 2236 & 7,04 & 2,68 & 2,76 & 12,48 \\
\hline 1997 & 2263 & 6,99 & 2,96 & 2,67 & 12,62 \\
\hline 1998 & 2290 & 6,77 & 2,69 & 2,67 & 12,13 \\
\hline
\end{tabular}

- Tab. 1 Fortsetzung

\begin{tabular}{|c|c|c|c|c|c|}
\hline \multirow{2}{*}{ Jahr } & \multirow{2}{*}{$\begin{array}{l}\text { Zigaretten- } \\
\text { äquivalente }\end{array}$} & \multicolumn{4}{|c|}{ Liter Reinalkohol } \\
\hline & & Bier & Wein & Spirituosen & Gesamt \\
\hline 1999 & 2380 & 6,75 & 2,97 & 2,56 & 12,28 \\
\hline 2000 & 2306 & 6,60 & 2,84 & 2,56 & 12,01 \\
\hline 2001 & 2360 & 6,43 & 2,96 & 2,53 & 11,92 \\
\hline 2002 & 2390 & 6,37 & 2,91 & 2,43 & 11,71 \\
\hline 2003 & 2250 & 6,14 & 3,39 & 2,34 & 11,87 \\
\hline 2004 & 2043 & 6,01 & 2,78 & 2,48 & 11,26 \\
\hline 2005 & 1948 & 5,92 & 2,96 & 2,30 & 11,19 \\
\hline 2006 & 1809 & 5,95 & 3,10 & 2,53 & 11,58 \\
\hline 2007 & 1812 & 5,71 & 3,06 & 2,47 & 11,24 \\
\hline 2008 & 1717 & 5,68 & 2,97 & 2,45 & 11,09 \\
\hline 2009 & 1687 & 5,57 & 3,23 & 2,28 & 11,08 \\
\hline 2010 & 1663 & 5,42 & 2,94 & $2,33^{c}$ & 10,69 \\
\hline 2011 & 1782 & 5,49 & 3,14 & 2,42 & 11,04 \\
\hline 2012 & 1689 & 5,35 & 3,18 & 2,45 & 10,98 \\
\hline 2013 & 1630 & 5,24 & 3,06 & 2,41 & 10,71 \\
\hline 2014 & 1621 & 5,25 & 2,85 & 2,47 & 10,58 \\
\hline \multicolumn{6}{|c|}{$\begin{array}{l}\text { a } 1 \text { Zigarette oder } 1 \text { Gramm Feinschnitt- oder Pfeifentabak oder 0,5 } \\
\text { Zigarre oder 0,5 Zigarillo; b Mittelwert aus den Jahren } 1988 \text { und } \\
\text { 1989; c Mittelwert aus den Jahren } 2009 \text { und 2011; Gesamt kann } \\
\text { Rundungsfehler enthalten }\end{array}$} \\
\hline
\end{tabular}

\section{Ergebnisse}

Der Tabakkonsum in Deutschland stieg von 1950 bis 1972 in einem Trend an ( Tab. 2, $\triangleright$ Abb. 1). Die höchste Zahl der Zigarettenäquivalente im Analysezeitraum betrug pro Bürgerin oder Bürger im Alter ab 15 Jahren 2919 ( $\triangleright$ Tab. 1). Das war im Jahr 1971. Der Tabakkonsum sank ab dem Jahr 1972 um 1,2 Prozentpunkte pro Jahr bis zum Jahr 2014, unterbrochen durch 2 kürzere Trends. Einer davon war eine Senkung des Konsums um jährlich 6,9 Prozentpunkte von 2002 bis 2006. Die Zahl der Zigarettenäquivalente im Jahr 2014 betrug 1621. Am Ende des gesamten Analysezeitraumes von 65 Jahren war der Tabakkonsum nicht niedriger als zu Beginn.

Der Alkoholverbrauch stieg seit 1950 in 3 einzelnen, jeweils schwächer werdenden Trends bis zum Jahr 1974 (\ Tab. 2, \ Abb. 2). Von 1974 bis zum Ende des Analysezeitraumes sank der Konsum mit jährlich 1,0 Prozentpunkten. Der höchste Konsum in den 65 Jahren betrug 16,7 Liter Reinalkohol pro Einwohnerin oder Einwohner im Alter ab 15 Jahren, im Jahr 2014 waren es 10,6 Liter. Am Ende des Analysezeitraumes von 65 Jahren war der Alkoholkonsum höher als zu Beginn.

\section{Diskussion}

2 Ergebnisse sind hervorzuheben. Erstens zeigen die Trendanalysen nach Anstiegen des Tabak- und des Alkoholkonsums moderate Konsumsenkungen. Zweitens fällt die Parallelität der beiden Konsumentwicklungen auf. 


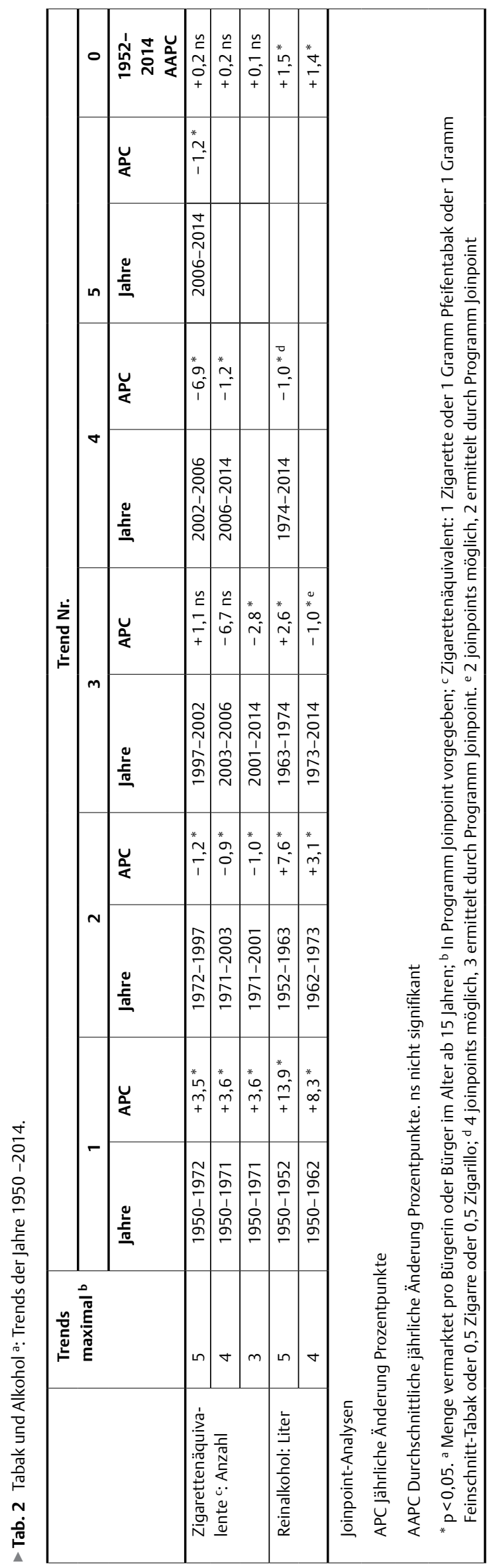

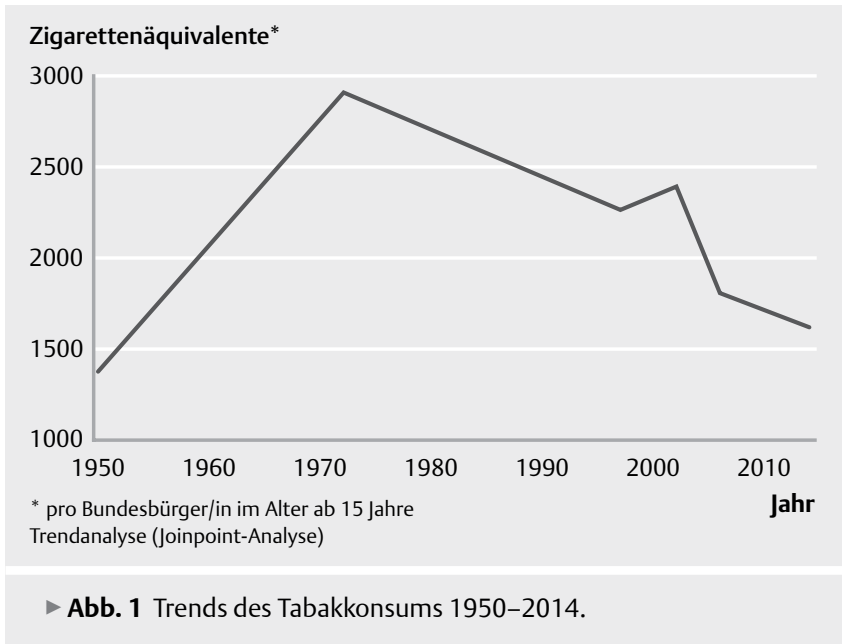

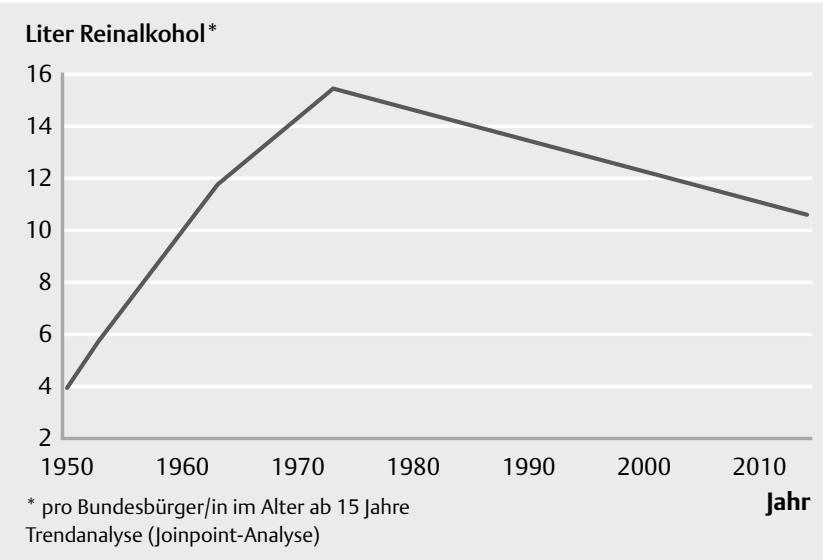

Abb. 2 Trends des Alkoholkonsums 1950-2014.

\section{Zeitliche Trends}

Der Tabakkonsum stieg bis zum Jahr 1972 und sank danach. Am Ende des Beobachtungszeitraumes im Jahr 2014 war er noch sehr hoch, 65 Jahre nach den ersten international anerkannten Publikationen zum Zusammenhang von Tabakrauchen und Krankheiten im Jahr 1950 [96]. Für Alkoholkonsum zeigen die Ergebnisse bis zum Jahr 1974 Anstiege, gefolgt von einer moderaten Senkung. Der Alkoholkonsum lag aber im Jahr 2014 noch über dem Niveau des Jahres 1960 und blieb auch im internationalen Vergleich sehr hoch.

Die Senkungen des Tabakrauchens folgen einem internationalen Trend der Reduktion altersstandardisierter Raten täglicher Raucher [97]. Dennoch wurde Deutschland als eine der 10 Nationen mit dem höchsten Tabakkonsum im Jahr 2014 gewertet [9]. In den USA stieg in den Jahren von 1950 bis 1976 die Zahl der Zigaretten pro Einwohner oder Einwohnerin von 2390 auf 2905. Danach sank sie auf 921 im Jahr 2012 [98], wenngleich zunehmend Zigarren und loser Tabak in den USA im Zeitraum 2002-2012 gekauft wurden [99]. In Kalifornien, einem besonders präventionsaktiven Staat, gab es nach Präventionsmaßnahmen noch stärkere Senkungen der Zahl der Zigaretten pro Einwohner oder Einwohnerin, sowohl im Vergleich zur Zeit vor Präventionsaktivitäten mit gesetzgeberischen Maßnah- 
men als auch im Vergleich zu den USA ohne Kalifornien [100]. In Deutschland war der Alkoholkonsum im Jahr 2014 noch so beträchtlich, dass die Bundesrepublik zu den 9 Ländern mit dem höchsten Konsum unter 40 Mitglieds- oder Partnerländern der OECD zählte [10]. Die Konsumänderungen bei Alkohol entsprechen einem Trend in Europa. In Frankreich, Griechenland, Italien, Portugal und Spanien sank der Alkoholverbrauch nach dem Höhepunkt von 14 Litern Reinalkohol pro Einwohnerin oder Einwohner im Jahr $1974[10,101]$.

Zu Erklärungen der beiden Trendkurven gehören Änderungen sozialer Normen. Sie prägen Verhaltensentscheidungen von Einzelpersonen. Soziale Normen des Konsums von Tabak und Alkohol werden insbesondere durch Gesetze und durch Förderung von Gesundheitsbewusstsein geändert [102]. Gesetzliche Regelungen sind international als besonders wirksamer Weg bewährt, Tabakrauchen und gesundheitsriskanten Alkoholkonsum unattraktiv zu machen. Gesetze können, müssen aber nicht mit dem Ziel der Prävention tabak- oder alkoholbezogener Krankheiten erlassen sein. Änderungen sozialer Normen finden u. a. aufgrund dreier gesellschaftlicher Veränderungen statt: einzelne gesetzliche Regelungen, ökonomische Gesichtspunkte sowie Schulbildung.

Gesetzliche Regelungen bilden ein zu bevorzugendes Instrument der Prävention tabak- und alkoholbezogener Krankheiten [103]. Gesetze können die Konsumsenkung beschleunigen, wie Befunde unserer Arbeit nahelegen. Die Intensivierung der Reduktion des Tabakkonsums von anfangs 1,2 Prozentpunkten auf 6,9 Prozentpunkte seit dem Jahr 2002 erscheint plausibel vor dem Hintergrund der Preisanhebungen ab dem Jahr 2002. Zigaretten wurden 2002 -2005 in mehreren Stufen um 51,8\% des Preises von 2001 verteuert [104] nach stabilen Zigarettenpreisen der Jahre 1990-2000 [105]. Ab dem Jahr 2002 erfolgten gesetzliche Maßnahmen des Jugendschutzes und der Einschränkung von Werbung für Tabakprodukte. Warnhinweise auf Zigarettenpackungen mussten vergröBert werden, 2004 wurden Arbeitnehmer am Arbeitsplatz vor Tabakrauch besser geschützt. Nach dem Jahr 2006 konnten weitere Maßnahmen die Stärke der Reduktion nicht aufrecht erhalten. Im Jahr 2007 wurde das Nichtraucherschutzgesetz in Bund und Ländern erlassen, 2007 erfolgte die Verbesserung des Jugendschutzes durch Anhebung der Lebensaltersgrenze zum Erwerb von Tabakwaren auf 18 Jahre. Automaten wurden mit einem Prüfsystem für Chip-Karten mit Informationen zum Lebensalter ausgerüstet, 2007 wurden Tabakwerbung in Printmedien und Internet verboten und Sponsoring von Sportveranstaltungen durch Tabakfirmen eingeschränkt $[12,106]$. Die Befunde ermutigen, diese Maßnahmen als Start für weitere Gesetzesinitiativen zu nehmen. Hinsichtlich der Präventionsaktivitäten zum Tabakrauchen im Jahr 2013 befand sich Deutschland unter 34 europäische Ländern auf dem vorletzten Rang [107].

Zur Alkoholkonsumsenkung können 2 gesetzliche Aktionsfelder der Prävention beigetragen haben: Maßnahmen zur Erhöhung der Verkehrssicherheit und ein Gesetz zum Schutz junger Menschen. Die Maßnahmen der Verkehrssicherheit umfassen Senkungen der zulässigen Blut-Alkohol-Konzentration (BAK). Sie können die Verringerung des Alkoholverbrauches gefördert haben, wenn man berücksichtigt, dass Autos zu einem alltäglichen Fortbewegungsmittel in der ganzen Bevölkerung wurden. Auch das berufliche Angewiesensein auf die Fahrerlaubnis kann die Gelegenheiten zum Alkoholkonsum verringert haben. Im Jahr 1960 waren in Deutschland 0,19, im Jahr 1970 0,38, im Jahr 1980 0,57, im Jahr 1990 0,69 und im Jahr 2000 0,77 Kraftfahrzeuge pro erwachsener Einwohner/in zugelassen [108]. Die Zahl der Straßenverkehrsunfälle, bei denen mind. eine der beteiligten Personen unter Alkoholeinfluss stand, verringerte sich von 25690 im Jahr 2001 auf 13612 in 2014 [109]. Im Jahr 1953 bestand die 1,5-Promille-Grenze BAK für absolute Fahruntüchtigkeit laut Strafgesetzbuch. Sie wurde 1966 auf 1,3 Promille gesenkt. Ab Juli 1973 galten 0,8-1,29 Promille BAK als Ordnungswidrigkeit mit Geldbußen bis 3000 DM und „im Regelfall“ 3 Monaten Fahrerlaubnis-Entzug. Ab 1,3 Promille BAK lag eine Straftat vor. Im Jahr 1998 wurde die zweistufige Promillegrenze eingeführt. Ab 0,5 Promille BAK lag eine Ordnungswidrigkeit vor, ab 0,8 Promille konnte Fahrverbot erteilt werden [110]. Am 1.4.2001 trat die 0,5-Promille-Grenze BAK in Kraft. Ab 1.8.2007 wurden 0,0 Promille bei Fahrern im Alter unter 21 Jahren sowie Fahranfängern in 2-jähriger Probezeit eingeführt [110]. Eine weitere, kleinere Maßnahme bildet die Verabschiedung des Gesetzes zur „Verbesserung des Schutzes junger Menschen vor den Gefahren des Alkohol- und Tabakkonsums“ im Mai 2004. Damit wurde eine Sondersteuer auf alkoholhaltige Süßgetränke („Alkopops“) erhoben, die der Branntweinsteuer unterlagen. Einnahmen aus dieser Alkopopsteuer werden der Bundeszentrale für gesundheitliche Aufklärung für Zwecke der Suchtprävention zugeführt. Insgesamt fehlt bei singulären Maßnahmen zur Erhöhung der Verkehrssicherheit und dem „Alkopops“-Gesetz gezieltes Engagement der Alkoholprävention in Deutschland. Die Ergebnisse der Senkung des Alkoholkonsums sprechen dafür, dass weitere Gesetzesänderungen aus der beträchtlichen Bandbreite möglicher Maßnahmen die Senkung des Konsums massiv stärken können. Für das Ziel der Verringerung des Alkoholkonsums fehlen in Deutschland Maßnahmen, die sich aufgrund von Evidenz bewährt haben. Dazu zählen Steuererhöhungen, Regelungen zu Zeiten und Orten der Erhältlichkeit von Alkohol sowie Jugendschutz [6, 111].

Zu ökonomischen Gesichtspunkten der Änderung sozialer Normen lässt sich für die 1950er und 1960er Jahre mit den gestiegenen Einkommen an besonders starken „Konsumhunger“ denken. Soziale Normen des Wohlstands bei hoher Quote beschäftigter Personen in der Bevölkerung schlossen hohen Alkoholkonsum ein. $\mathrm{Er}$ lässt Beeinträchtigungen von Leistungen in der Arbeitswelt erwarten. Im Zuge von Wirtschaftskrisen könnten insbesondere diejenigen Arbeitnehmer ihre Arbeitsstelle verlieren [112], die am meisten Alkohol konsumieren. Dieser Annahme entsprechen Zusammenhänge des Rückgangs des Alkoholkonsums mit Anstiegen der Arbeitslosenrate. Nach einem historischen Tiefststand von 0,7\% im Jahr 1970 stieg sie an, im Jahr 1983 betrug sie 9,1 und im Jahr 2005 13,0 \% [113]. In Spanien wurden ähnliche Zusammenhänge gefunden [101].

Schulbildung kann grundsätzlich die Reduktion von Tabak- und Alkoholkonsum fördern. Evidenz zeigt, dass die Raucherrate umso höher ist, je niedriger die Schulbildung ausfällt [114-116]. Gesundheitsriskanter Alkoholkonsum scheint bei Männern mit niedriger Schulbildung prävalenter zu sein als bei Männern mit höherer Schulbildung [5, 117]. Alkohol-attributable Mortalität, begrenzt auf Erkrankungen, die Alkoholkonsum voraussetzen, weist bei Männern und Frauen mit geringer Bildung eine höhere Prävalenz auf als bei Männern und Frauen mit hoher Bildung [118]. Diese soziale Dis- 
paratheit ist bei alkohol-attributabler Mortalität ausgeprägter als bei allgemeiner Mortalität [119]. Die Schulbildung stieg in Deutschland an. In der jeweils 19 Jahre alten Geburtskohorte betrug der Anteil derer, die Abitur als Schulabschluss aufwiesen, bei Frauen 3,1 \% im Jahr 1950, 4,3\% im Jahr 1960, 8,2\% im Jahr 1970, 15,4\% im Jahr 1980 und 35,3\% im Jahr 2010. Unter Männern betrug der Anteil 6,1 \% im Jahr 1950, 7,4\% im Jahr 1960, 12,1 \% im Jahr 1970, 15,6\% im Jahr 1980 und 27,0\% im Jahr 2010 [120, 121]. Mögliche Einflüsse der Schulbildung auf die Senkung von Raucherraten sind historisch auch vor dem Hintergrund des Entstehens von Wissen um die Gefährlichkeit des Tabakrauchens für die Gesundheit seit 1950 zu betrachten.

\section{Parallelität der Trends des Tabak- und des Alkoholkonsums}

Die Parallelität der Trends des Tabak- und Alkoholkonsums in Deutschland stützt die Annahme einer gegenseitigen Verstärkung der Reduktionen des Tabak- sowie des Alkoholkonsums. Das steht auch im Einklang mit weiteren Befunden. So waren Preiserhöhungen bei Tabakprodukten und Gesetze zum Schutz der Atemluft vor Tabakrauch von Reduktionen des Alkoholkonsums gefolgt [7].

Die Ergebnisse legen nahe, dass der Tabakkonsum nach dem Jahr 2000 eine stärkere Reduktion erfahren hat als der Alkoholkonsum. Bei Betrachtung der Joinpoint-Analysen mit 2 Trends zeigt der Tabakkonsum in den Jahren 1971-2001 eine durchschnittliche jährliche Senkung von einem Prozentpunkt. Sie wurde jedoch in den Jahren 2001 bis zum Ende des Beobachtungszeitraumes auf 2,8 Prozentpunkte pro Jahr fast verdreifacht. Bei dem Alkoholkonsum beträgt die Senkung von 1973 bis zum Ende des Beobachtungszeitraumes einen Prozentpunkt pro Jahr. Die stärkere Senkung des Tabakkonsums erscheint durch die dargestellten Leistungen der Prävention tabakbezogener Schäden seit dem Jahr 2002 besonders plausibel im Vergleich zur moderateren Senkung des Alkoholkonsums.

Die Trends zu Senkungen werden durch Surveydaten unterstützt. Daten des Mikrozensus in Deutschland zeigen 36,7\% der Männer und 17,9\% der Frauen im Alter ab 35 Jahren als gegenwärtige Raucher im Jahr 1989 und 27,5\% der Männer sowie 18,9\% der Frauen im Alter ab 35 Jahren im Jahr 2009 als gegenwärtige Raucher [122]. Der Anteil derjenigen, die das Rauchen beendet hatten, war über den Zeitraum gestiegen. Auch 9 wiederholte Querschnittbefragungen mit Stichproben Erwachsener in unterschiedlichen Lebensaltersbereichen bis zu 64 Jahren zeigten im Rahmen des Epidemiologischen Suchtsurveys in den Jahren 1980-2009 eine Senkung derer, die angaben in den letzten 30 Tagen vor der Befragung Zigaretten geraucht zu haben, sowie eine Senkung der Zahl der gerauchten Zigaretten [123]. Bei 18- bis 59-jährigen Surveyteilnehmern ergab sich in den Jahren 1995-2015 ein Trend zur Verringerung der Personen, die angaben, in den letzten 30 Tagen vor der Befragung Alkohol getrunken zu haben [124].

\section{Vorzüge und Limitationen}

Die Befunde bieten besonders valide Schätzungen des Tabak- und des Alkoholkonsums der Bundesrepublik Deutschland seit dem Jahr 1950. Die durchgeführten Datenanalysen ermöglichen ein Monitoring von 2 der bedeutendsten Outcome-Merkmale der Prävention verbreiteter Krankheiten und weitere Analysen. Plausibel wird durch die Befunde z. B., unterstützt durch Erfahrungen aus anderen Ländern, dass ab 1976 die Leberzirrhosemortalität sank [125]. Bei diesen Vorzügen sind aber auch 5 Limitationen der Ergebnisse zu berücksichtigen.

Unsere Schätzungen des Alkoholkonsums können aus 3 Gründen nur als Mindestmengen des wahren Alkoholkonsums gelten: 1. Bei Bier haben wir für die Jahre 1950-1992 aufgrund fehlender Differenzierung der Daten pauschal einen Alkoholgehalt von 4,8 Volumen-\% eingesetzt. Das entspricht etwa der unteren Grenze der Stammwürze-Angaben 11-14 Grad Plato, die für die Jahre vor 1993 veröffentlicht und in der mehr als $98 \%$ der versteuerten Biermengen geführt sind. 2. Seit dem Jahr 1993 verblieb für die Biere mit dem höchsten Alkoholgehalt ebenfalls eine Gruppe („22-35“ oder „22 und darüber“), für die wir die untere Grenze (22 Grad Plato) werteten. 3. Der Alkoholgehalt von Wein ist empirisch nur durch Öchsle-Grade für inländisch hergestellten Wein geschätzt. Für importierten Wein war das nicht möglich. Importierter Wein hatte im Jahre 2014 auf dem Markt in Deutschland einen Anteil von $55 \%$ [126], $37 \%$ aus Frankreich, Italien oder Spanien und $18 \%$ aus weiteren Ländern. Die hohe Sonneneinstrahlung in diesen Ländern lässt einen höheren Alkoholgehalt als bei deutschen Weinen erwarten. Auch das macht eine Unterschätzung der konsumierten Alkoholmengen wahrscheinlich. 4. In den Trinkweinmengen der Jahre 1964-2014 sind Erzeugnisse enthalten, denen Destillationsalkohol zugesetzt wurde, z. B. Portwein (19-22 Volumen-\%), Sherry (17\%) und Madeira (18-21\%) mit Alkoholgehalten bis 22 Volumen-\% Alkohol [127]. Der Anteil dieser Erzeugnisse an der Gesamtmenge an Wein liegt aber nur bei 1\%. 5. Geringfügige Ungenauigkeiten der Schätzungen können durch Korrekturen der Bevölkerungszahlen Deutschlands entstanden sein, zuletzt aufgrund des Zensus 2011. Über diese 5 Limitationen hinaus unterliegt die Analyse den Begrenzungen der Vermarktungsdaten. Mögliche Einflüsse durch Lebensalter und Geburtskohorten konnten nicht geprüft werden.

\section{FAZIT}

1. Tabakrauchen und Alkoholkonsum zeigen zeitgleiche Trends des Anstiegs bis in die erste Hälfte der 1970er Jahre und danach moderate Senkungen.

2. Nach Inkrafttreten präventionsrelevanter Gesetze zeigten sich Verstärkungen des Trends zur Senkung von Tabakkonsum.

3. Der Konsum von Tabak und Alkohol ist 65 Jahre nach den ersten international anerkannten Publikationen zum Zusammenhang zwischen Tabakrauchen und Erkrankungsrisiko im internationalen Vergleich in Deutschland sehr hoch, trotz der Senkungen seit 1972.

4. Ausweitungen präventionsrelevanter gesetzlicher Maßnahmen versprechen weitere Senkungen des Tabak- und Alkoholkonsums. Die lassen Erhöhungen der Lebenserwartung der Bevölkerung Deutschlands erwarten. 


\section{Danksagung}

Wir danken den Mitarbeiterinnen und Mitarbeitern mehrerer Abteilungen des Statistischen Bundesamtes, v. a. aus dem Bereich Verbrauchsteuer, für die Bereitstellung umfangreicher Daten und die sehr hilfreichen Auskünfte. Diese Unterstützung trug maßgeblich zum Gelingen der Arbeit bei.

Interessenkonflikt

Die Autoren geben an, dass sie kein Interessenkonflikt besteht.

\section{Literatur}

[1] John U, Ulbricht S, Freyer-Adam J et al. Verhaltensbasierte Prävention chronischer Krankheiten. Dtsch Med Wochenschr. 2015; 140: 756-760

[2] Leifman H, Österberg E, Ramstedt M. Alcohol in postwar Europe: a discussion of indicators on consumption and alcohol-related harm.Stockholm: 2002 [zugegriffen am: 24.03.2016] http://ec. europa.eu/health/ph_projects/1998/monitoring/fp_monitoring_1998_frep_01_a_en.pdf

[3] Cnossen S. Alcohol taxation and regulation in the European Union. Int Tax Public Finan 2007; 14: 699-732

[4] Adams M, Effertz T. Effective prevention against risky underage drinking-the need for higher excise taxes on alcoholic beverages in Germany. Alcohol Alcohol 2010; 45: 387-394

[5] Devaux M, Sassi F. Social disparities in hazardous alcohol use: self-report bias may lead to incorrect estimates. Eur J Public Health 2016; 26: 129-134

[6] John U, Meyer C, Ulbricht S et al. Reduktion von Tabak- und Alkoholkonsum. In: Hoefert W, Klauer T. Krankheitsprävention in der Kontroverse. Lengerich: Pabst; 2014: S. 307-325

[7] Krauss MJ, Cavazos-Rehg PA, Plunk AD et al. Effects of state cigarette excise taxes and smoke-free air policies on state per capita alcohol consumption in the United States, 1980 to 2009. Alcohol Clin Exp Res 2014; 38: 2630-2638

[8] Guydish J, Passalacqua E, Pagano A et al. An international systematic review of smoking prevalence in addiction treatment. Addiction 2016; 111: 220-230

[9] Eriksen M, Mackay J, Schluger $\mathrm{N}$ et al. The Tobacco Atlas. Atlanta, Georgia: American Cancer Society; 2015

[10] OECD. Tackling harmful alcohol use: economics and public health policy. OECD Publishing; 2015 [zugegriffen am: 20.07.2016] http// dx.doi.org/10.1787/9789264181069-en

[11] John U, Hanke M, Meyer C et al. Alkohol. In: Deutsche Hauptstelle für Suchtfragen, Jahrbuch Sucht 2016. Lengerich: Pabst; 2016. S. 37-54

[12] Lampert T, Kuntz B. Tabak - Zahlen und Fakten zum Konsum. In: Deutsche Hauptstelle für Suchtfragen, Jahrbuch Sucht 2015. Lengerich: Pabst; 2015: S. 72-101

[13] OECD. OECD health statistics 2015 definitions, sources and methods. Alcohol consumption in liters per capita (age 15+). OECD Publishing; 2015 [zugegriffen am: 20.07.2016] http://www.oecd.org/health/ health-data.htm

[14] Bühringer G, Augustin R, Bergmann E et al. Alkoholkonsum und alkoholbezogene Störungen in Deutschland. Baden-Baden: Nomos; 2000

[15] Statistisches Bundesamt. Annähernder Verbrauch von Tabakwaren, Tabellen für die Jahre 1950 bis 1990. Wiesbaden: Statistisches Bundesamt; 2014
[16] Statistisches Bundesamt. Finanzen und Steuern. Arbeitsunterlage zu den Verbrauchssteuerstatistiken. Zeitreihe für die Berichtsjahre 1991 bis 2004. Verwendete Daten: Tabak: Jahre 1991-1999, Bier: 1991-1992, Wein und Spirituosen: 1991-1999.Wiesbaden: 2005 [zugegriffen am: 16.02.2017] https://www.destatis.de/GPStatistik/ receive/DEHeft_heft_00023242

[17] Statistisches Bundesamt. Finanzen und Steuern. Arbeitsunterlage zu den Verbrauchssteuerstatistiken. Zeitreihe für die Berichtsjahre 2000 bis 2015. Verwendete Daten: Tabak, Wein, Spirituosen: Jahre 2000 bis 2014.Wiesbaden: 2016; [zugegriffen am: 16.02.2017] https:// www.destatis.de/DE/Publikationen/Thematisch/FinanzenSteuern/ Steuern/Verbrauchsteuer/Verbrauchsteuerstatistik.html

[18] OECD. OECD Health Statistics 2014. Definitions, Sources and Methods. Tobacco consumption in grams per capita (age 15+). 2014; [zugegriffen am: 15.08. 2014] http://www.oecd.org/els/ health-systems/health-data.htm

[19] Statistisches Bundesamt.. Fachserie 14, Reihe 9.2.1 Finanzen und Steuern. Absatz von Bier 1994, Verwendete Daten: Jahr 1993. Wiesbaden: 1995

[20] Statistisches Bundesamt.. Fachserie 14, Reihe 9.2.1 Finanzen und Steuern. Absatz von Bier 1995, Verwendete Daten: Jahr 1994. Wiesbaden: 1996

[21] Statistisches Bundesamt. Fachserie 14, Reihe 9.2.1 Finanzen und Steuern. Absatz von Bier 1996, Verwendete Daten: Jahr 1995. Wiesbaden: 1997

[22] Statistisches Bundesamt. Fachserie 14, Reihe 9.2.1 Finanzen und Steuern. Absatz von Bier 1997, Verwendete Daten: Jahr 1996. Wiesbaden: 1998

[23] Statistisches Bundesamt. Fachserie 14, Reihe 9.2.1 Finanzen und Steuern. Absatz von Bier 1998, Verwendete Daten: Jahr 1997. Wiesbaden: 1999

[24] Statistisches Bundesamt. Fachserie 14, Reihe 9.2.1 Finanzen und Steuern. Absatz von Bier Dezember 1999, Verwendet Daten: Jahr 1998. Wiesbaden: 2000

[25] Statistisches Bundesamt. Fachserie 14, Reihe 9.2.1 Finanzen und Steuern. Absatz von Bier Dezember 2000, Verwendete Daten: Jahr 1999. Wiesbaden: 2001

[26] Statistisches Bundesamt. Fachserie 14, Reihe 9.2.1 Finanzen und Steuern. Absatz von Bier, Dezember 2001, Verwendete Daten: Jahr 2000. Wiesbaden: 2002

[27] Statistisches Bundesamt. Fachserie 14, Reihe 9.2.1 Finanzen und Steuern. Absatz von Bier, Dezember 2002, Verwendete Daten: Jahr 2001. Wiesbaden: 2003

[28] Statistisches Bundesamt. Fachserie 14, Reihe 9.2.1 Finanzen und Steuern. Absatz von Bier, Dezember 2003, Verwendete Daten: Jahr 2002. Wiesbaden: 2004

[29] Statistisches Bundesamt. Fachserie 14, Reihe 9.2.2 Finanzen und Steuern, Brauwirtschaft 2003, Tabelle „Über Zollstellen versteuertes Bier aus Drittländern“, Verwendete Daten: 2002. Wiesbaden: 2004 [zugegriffen am: 17.02.2017] https://www.destatis.de/GPStatistik/ receive/DEHeft_heft_00014241

[30] Statistisches Bundesamt. Fachserie 14, Reihe 9.2.1 Finanzen und Steuern. Absatz von Bier, Dezember 2004, Verwendete Daten: Jahr 2003. Wiesbaden: 2005 [zugegriffen am: 17.02.2017] https://www. destatis.de/GPStatistik/receive/DEHeft_heft_00013995

[31] Statistisches Bundesamt. Fachserie 14, Reihe 9.2.2 Finanzen und Steuern, Brauwirtschaft 2004, Tabelle „Über Zollstellen versteuertes Bier aus Drittländern“, Verwendete Daten: 2003. Wiesbaden. 2005; [zugegriffen am: 17.02.2017] https://www.destatis.de/GPStatistik/ receive/DEHeft_heft_00021474

[32] Statistisches Bundesamt. Fachserie 14, Reihe 9.2.1 Finanzen und Steuern. Absatz von Bier, Dezember und Jahr 2005, Verwendete Daten: Jahr 2004. Wiesbaden. 2006; [zugegriffen am: 17.02.2017] https://www.destatis.de/GPStatistik/receive/DEHeft_heft_00014031 
[33] Statistisches Bundesamt. Fachserie 14, Reihe 9.2.2 Finanzen und Steuern, Brauwirtschaft 2005, Tabelle „Über Zollstellen versteuertes Bier aus Drittländern“, Verwendete Daten: 2004. Wiesbaden. 2006; [zugegriffen am: 17.02.2017] https://www.destatis.de/GPStatistik/ receive/DEHeft_heft_00021477

[34] Statistisches Bundesamt. Fachserie 14, Reihe 9.2.1 Finanzen und Steuern. Absatz von Bier, Dezember und Jahr 2006, Verwendete Daten: Jahr 2005. Wiesbaden. 2007; [zugegriffen am: 17.02.2017] https://www.destatis.de/GPStatistik/receive/DEHeft_heft_00014067

[35] Statistisches Bundesamt. Fachserie 14, Reihe 9.2.2 Finanzen und Steuern, Brauwirtschaft 2006, Tabelle „Über Zollstellen versteuertes Bier aus Drittländern“, Verwendete Daten: 2005. Wiesbaden. 2007; [zugegriffen am: 17.02.2017] https://www.destatis.de/GPStatistik/ receive/DEHeft_heft_00021480

[36] Statistisches Bundesamt. Fachserie 14, Reihe 9.2.1 Finanzen und Steuern. Absatz von Bier, Dezember und Jahr 2007, Verwendete Daten: Jahr 2006. Wiesbaden. 2008; [zugegriffen am: 17.02.2017] https://www.destatis.de/GPStatistik/receive/DEHeft_heft_00014103

[37] Statistisches Bundesamt. Fachserie 14, Reihe 9.2.2 Finanzen und Steuern, Brauwirtschaft 2007, Tabelle „Über Zollstellen versteuertes Bier aus Drittländern“, Verwendete Daten: 2006. Wiesbaden. 2008; [zugegriffen am: 17.02.2017] https://www.destatis.de/GPStatistik/ receive/DEHeft_heft_00014244

[38] Statistisches Bundesamt. Fachserie 14, Reihe 9.2.1 Finanzen und Steuern. Absatz von Bier, Dezember und Jahr 2008, Verwendete Daten: Jahr 2007. Wiesbaden. 2009; [zugegriffen am: 17.02.2017] https://www.destatis.de/GPStatistik/receive/DEHeft_heft_00014139

[39] Statistisches Bundesamt. Fachserie 14, Reihe 9.2.2 Finanzen und Steuern, Brauwirtschaft 2008, Tabelle „Über Zollstellen versteuertes Bier aus Drittländern“, Verwendete Daten: 2007. Wiesbaden. 2009; [zugegriffen am: 17.02.2017] https://www.destatis.de/GPStatistik/ receive/DEHeft_heft_00014248

[40] Statistisches Bundesamt. Fachserie 14, Reihe 9.2.1 Finanzen und Steuern. Absatz von Bier, Dezember und Jahr 2009, Verwendete Daten: Jahr 2008. Wiesbaden. 2010; [zugegriffen am: 17.02.2017] https://www.destatis.de/GPStatistik/receive/DEHeft_heft_00014172

[41] Statistisches Bundesamt. Fachserie 14, Reihe 9.2.2 Finanzen und Steuern, Brauwirtschaft 2009, Tabelle „Über Zollstellen versteuertes Bier aus Drittländern“, Verwendete Daten: 2008. Wiesbaden. 2010; [zugegriffen am: 17.02.2017] https://www.destatis.de/GPStatistik/ receive/DEHeft_heft_00014251

[42] Statistisches Bundesamt. Fachserie 14, Reihe 9.2.1 Finanzen und Steuern. Absatz von Bier, Dezember und Jahr 2010, Verwendete Daten: Jahr 2009. Wiesbaden. 2011; [zugegriffen am: 17.02.2017] https://www.destatis.de/GPStatistik/receive/DEHeft_heft_00014210

[43] Statistisches Bundesamt. Fachserie 14, Reihe 9.2.2 Finanzen und Steuern, Brauwirtschaft 2010, Tabelle „Über Zollstellen versteuertes Bier aus Drittländern“, Verwendete Daten: 2009. Wiesbaden. 2011; [zugegriffen am: 17.02.2017] https://www.destatis.de/GPStatistik/ receive/DEHeft_heft_00014254

[44] Statistisches Bundesamt. Fachserie 14, Reihe 9.2.1 Finanzen und Steuern. Absatz von Bier, Dezember und Jahr 2011, Verwendete Daten: Jahr 2010. Wiesbaden. 2012; [zugegriffen am: 17.02.2017] https://www.destatis.de/GPStatistik/receive/DEHeft_heft_00016522

[45] Statistisches Bundesamt. Fachserie 14, Reihe 9.2.2 Finanzen und Steuern, Brauwirtschaft 2011, Tabelle „Über Zollstellen versteuertes Bier aus Drittländern“, Verwendete Daten: 2010. Wiesbaden. 2012; [zugegriffen am: 17.02.2017] https://www.destatis.de/GPStatistik/ receive/DEHeft_heft_00016440

[46] Statistisches Bundesamt. Fachserie 14, Reihe 9.2.1 Finanzen und Steuern. Absatz von Bier, Dezember und Jahr 2012, Verwendete Daten: Jahr 2011. Wiesbaden. 2013; [zugegriffen am: 17.02.2017] https://www.destatis.de/GPStatistik/receive/DEHeft_heft_00016434
[47] Statistisches Bundesamt. Fachserie 14, Reihe 9.2.2 Finanzen und Steuern, Brauwirtschaft 2012, Tabelle „Über Zollstellen versteuertes Bier aus Drittländern“, Verwendete Daten: 2011. Wiesbaden. 2013; [zugegriffen am: 17.02.2017] https://www.destatis.de/GPStatistik/ receive/DEHeft_heft_00018645

[48] Statistisches Bundesamt. Fachserie 14, Reihe 9.2.1 Finanzen und Steuern. Absatz von Bier, Dezember und Jahr 2013, Verwendete Daten: Jahr 2012. Wiesbaden. 2014; [zugegriffen am: 17.02.2017] https://www.destatis.de/GPStatistik/receive/DEHeft_heft_00021931

[49] Statistisches Bundesamt. Fachserie 14, Reihe 9.2.2 Finanzen und Steuern, Brauwirtschaft 2013, Tabelle „Über Zollstellen versteuertes Bier aus Drittländern“, Verwendete Daten: 2012. Wiesbaden. 2014; [zugegriffen am: 17.02.2017] https://www.destatis.de/GPStatistik/ receive/DEHeft_heft_00022189

[50] Statistisches Bundesamt. Fachserie 14, Reihe 9.2.1 Finanzen und Steuern. Absatz von Bier, Dezember und Jahr 2014, Verwendete Daten: Jahr 2013. Wiesbaden. 2015; [zugegriffen am: 17.02.2017] https://www.destatis.de/GPStatistik/receive/DEHeft_heft_00029721

[51] Statistisches Bundesamt. Fachserie 14, Reihe 9.2.2 Finanzen und Steuern, Brauwirtschaft 2014, Tabelle „Über Zollstellen versteuertes Bier aus Drittländern“, Verwendete Daten: 2013. Wiesbaden. 2015; [zugegriffen am: 17.02.2017] https://www.destatis.de/GPStatistik/ receive/DEHeft_heft_00029691

[52] Statistisches Bundesamt. Fachserie 14, Reihe 9.2.1 Finanzen und Steuern. Absatz von Bier, Dezember und Jahr 2015, Verwendete Daten: Jahr 2014. Wiesbaden. 2016; [zugegriffen am: 17.02.2017] https://www.destatis.de/GPStatistik/receive/DEHeft_heft_00044574

[53] Statistisches Bundesamt. Über Zollstellen versteuertes Bier aus Drittländern, Jahre 1993 bis 2001. Wiesbaden: Statistisches Bundesamt; 2016

[54] Statistisches Bundesamt. Fachserie 14, Reihe 9.2.2 Finanzen und Steuern, Brauwirtschaft 2015, Tabelle „Über Zollstellen versteuertes Bier aus Drittländern“, Verwendete Daten: 2014. Wiesbaden. 2016; [zugegriffen am: 17.02.2017] https://www.destatis.de/GPStatistik/ receive/DEHeft_heft_00044582

[55] Statistisches Bundesamt. Annähernder Verbrauch alkoholischer Getränke, Tabellen für die Jahre 1950 bis 1990, Verwendete Daten: Bier und Spirituosen 1950-1990, Wein 1964-1990. Wiesbaden: Statistisches Bundesamt; 2014

[56] Bundesministerium für Ernährung Landwirtschaft und Forsten. Versorgung mit Bier und Wein in $1000 \mathrm{hl}$. Kapitel C. Versorgungslage, VII Sonstige Nahrungsmittel, Verwendete Daten: Wein Jahre 1950 und 1952. In: Bundesministerium für Ernährung LuF. Statistisches Handbuch über Landwirtschaft und Ernährung der Bundesrepublik Deutschland 1956. Hamburg und Berlin: Verlag Paul Parey; 1956: S. 178 [zugegriffen am: 20.02.2017] http://www.bmel-statistik.de/ service/archiv/statistisches-jahrbuch/

[57] Bundesministerium für Ernährung Landwirtschaft und Forsten. Versorgung mit Bier und Wein in $1000 \mathrm{hl}$. Kapitel IX. Alkoholische Getränke, Genußmittel, Verwendete Daten: Wein Jahre 1951, 1953-1958. In: Bundesministerium für Ernährung LuF. Statistisches Jahrbuch über Ernährung, Landwirtschaft und Forsten 1959. Hamburg und Berlin: Verlag Paul Parey; 1959: S. 186

[58] Bundesministerium für Ernährung Landwirtschaft und Forsten. Versorgung mit Bier und Wein. Kapitel IX. Alkoholische Getränke, Genußmittel, Verwendete Daten: Wein Jahre 1959-1963. In: Bundesministerium für Ernährung LuF. Statistisches Jahrbuch über Ernährung, Landwirtschaft und Forsten 1965. Hamburg und Berlin: Verlag Paul Parey; 1965: S. 200 
[59] Statistisches Bundesamt. Weinbau und Weinmosternte, Kapitel IX Land- und Forstwirtschaft, Fischerei, Teil B. Bodennutzung und Ernte, Tabelle 9, Verwendete Daten: Jahre 1951-1953, 1955-1957. In: Statistisches Bundesamt. Statistisches Jahrbuch für die Bundesrepublik Deutschland 1961. Wiesbaden: Verlag Kohlhammer, Stuttgart und Mainz; 1961: S. 178 [zugegriffen am: 17.02.2017] http://www. digizeitschriften.de/dms/toc/?PID = PPN514402342_1961

[60] Statistisches Bundesamt. Rebfläche und Weinmosternte, Kapitel IX Land- und Forstwirtschaft, Fischerei - C. Bodennutzung und Ernte, Tabelle 7, Verwendete Daten: Jahre 1958 bis 1964. In: Statistisches Bundesamt. Statistisches Jahrbuch für die Bundesrepublik Deutschland 1966. Wiesbaden: Verlag Kohlhammer, Stuttgart und Mainz; 1966: S. 190 [zugegriffen am: 17.02.2017] http://www.digizeitschriften.de/dms/toc/?PID = PPN514402342_1966

[61] Statistisches Bundesamt. Anbau von Reben und Weinmosternte, Kapitel X Land- und Forstwirtschaft, Fischerei - C. Bodennutzung und Ernte, Tabelle 9, Verwendete Daten: Jahre 1965 bis 1968. In: Statistisches Bundesamt. Statistisches Jahrbuch für die Bundesrepublik Deutschland 1969. Wiesbaden: Verlag Kohlhammer, Stuttgart und Mainz; 1969: S. 161[zugegriffen am: 20.02.2017] http://www. digizeitschriften.de/dms/toc/?PID = PPN514402342_1969

[62] Statistisches Bundesamt. Anbau von Reben und Weinmosternte, Kapitel X Land- und Forstwirtschaft, Fischerei - C. Bodennutzung und Ernte, Tabelle 8, Verwendete Daten: Jahre 1969 bis 1970. In: Statistisches Bundesamt. Statistisches Jahrbuch für die Bundesrepublik Deutschland 1972. Wiesbaden: Verlag Kohlhammer, Stuttgart und Mainz; 1972: S. 154 [zugegriffen am: 20.02.2017] http://www. digizeitschriften.de/dms/toc/?PID = PPN514402342_1972

[63] Statistisches Bundesamt. Anbau von Reben und Weinmosternte, Kapitel X Land- und Forstwirtschaft, Fischerei - C. Bodennutzung und Ernte, Tabelle 8, Verwendete Daten: Jahre 1971-1972. In: Statistisches Bundesamt. Statistisches Jahrbuch für die Bundesrepublik Deutschland 1974. Wiesbaden: Verlag Kohlhammer, Stuttgart und Mainz; 1974: S. 176 [zugegriffen am: 20.02.2017] http://www. digizeitschriften.de/dms/toc/?PID = PPN514402342_1974

[64] Statistisches Bundesamt. Weinmosternte, Kapitel 13 Land- und Forstwirtschaft, Fischerei, Tabelle 13.29, Verwendete Daten: Jahre 1973-1974.In: Statistisches Bundesamt. Statistisches Jahrbuch 1976 für die Bundesrepublik Deutschland. Wiesbaden: Verlag Kohlhammer, Stuttgart und Mainz; 1976: S. 221[zugegriffen am: 20.02.2017] http://www.digizeitschriften.de/dms/

toc/?PID = PPN514402342_1976

[65] Statistisches Bundesamt. Weinmosternte, Kapitel 8 Land- und Forstwirtschaft, Fischerei, Tabelle 8.21, Verwendete Daten: Jahre 1975-1976.In: Statistisches Bundesamt. Statistisches Jahrbuch 1978 für die Bundesrepublik Deutschland. Wiesbaden: Verlag Kohlhammer, Stuttgart und Mainz; 1978: S. 150 [zugegriffen am: 20.02.2017] http://www.digizeitschriften.de/dms/toc/?PID = PPN51 4402342_1978

[66] Statistisches Bundesamt. Weinmosternte, Kapitel 8 Land- und Forstwirtschaft, Fischerei, Tabelle 8.20, Verwendete Daten: Jahre 1977-1978. In: Statistisches Bundesamt. Statistisches Jahrbuch 1980 für die Bundesrepublik Deutschland. Wiesbaden: Verlag Kohlhammer, Stuttgart und Mainz; 1980: S. 148 [zugegriffen am: 20.02.2017] http://www.digizeitschriften.de/dms/ toc/?PID = PPN514402342_1980

[67] Statistisches Bundesamt. Weinmosternte, Kapitel 8 Land- und Forstwirtschaft, Fischerei, Tabelle 8.25, Verwendete Daten: Jahre 1979-1980.In: Statistisches Bundesamt. Statistisches Jahrbuch 1982 für die Bundesrepublik Deutschland.Wiesbaden: Verlag Kohlhammer, Stuttgart und Mainz; 1982: S. 153 [zugegriffen am: 20.02.2017] http://www.digizeitschriften.de/dms/ toc/?PID = PPN514402342_1982
[68] Statistisches Bundesamt. Weinmosternte, Kapitel 8 Land- und Forstwirtschaft, Fischerei, Tabelle 8.23, Verwendete Daten: Jahre 1981-1982.In: Statistisches Bundesamt. Statistisches Jahrbuch 1984 für die Bundesrepublik Deutschland.Wiesbaden: Verlag Kohlhammer, Stuttgart und Mainz; 1984: S. 158 [zugegriffen am: 20.02.2017] http://www.digizeitschriften.de/dms/ toc/?PID = PPN514402342_1984

[69] Statistisches Bundesamt. Weinmosternte, Kapitel 8 Land- und Forstwirtschaft, Fischerei, Tabelle 8.24, Verwendete Daten: Jahre 1983-1984. In: Statistisches Bundesamt. Statistisches Jahrbuch 1986 für die Bundesrepublik Deutschland. Wiesbaden: Verlag Kohlhammer, Stuttgart und Mainz; 1986: S. 156 [zugegriffen am: 20.02.2017] http://www.digizeitschriften.de/dms/toc/?PID = PPN514 402342_1986

[70] Statistisches Bundesamt. Weinmosternte, Kapitel 8 Land- und Forstwirtschaft, Fischerei, Tabelle 8.22, Verwendete Daten: Jahre 1985-1986. In: Statistisches Bundesamt. Statistisches Jahrbuch 1988 für die Bundesrepublik Deutschland.Wiesbaden: Verlag Kohlhammer, Stuttgart und Mainz; 1988: S. 152 [zugegriffen am: 20.02.2017] http://www.digizeitschriften.de/dms/ toc/?PID = PPN514402342_1988

[71] Statistisches Bundesamt. Weinmosternte, Kapitel 8 Land- und Forstwirtschaft, Fischerei, Tabelle 8.26, Verwendete Daten: Jahr 1987. In: Statistisches Bundesamt. Statistisches Jahrbuch für die Bundesrepublik Deutschland 1990. Wiesbaden: Verlag MetzlerPoeschel, Stuttgart; 1990: S. 163 [zugegriffen am: 20.02.2017] http://www.digizeitschriften.de/dms/ toc/?PID = PPN514402342_1990

[72] Statistisches Bundesamt. Weinmosternte, Kapitel 8 Land- und Forstwirtschaft, Fischerei, Tabelle 8.24, Verwendete Daten: Jahre 1988-1990. In: Statistisches Bundesamt. Statistisches Jahrbuch 1991 für das vereinte Deutschland. Wiesbaden: Verlag Metzler-Poeschel, Stuttgart; 1991: S. 179 [zugegriffen am: 20.02.2017] http://www. digizeitschriften.de/dms/toc/?PID = PPN635628112_1991

[73] Statistisches Bundesamt. Weinmosternte, Kapitel 8 Land- und Forstwirtschaft, Fischerei, Tabelle 8.24, Verwendete Daten: Jahre 1991-1992. In: Statistisches Bundesamt. Statistisches Jahrbuch 1993 für die Bundesrepublik Deutschland. Wiesbaden: Verlag MetzlerPoeschel, Stuttgart; 1993: S. 188, http://www.digizeitschriften.de/ $\mathrm{dms} /$ toc/?PID = PPN635628112_1993

[74] Statistisches Bundesamt. Weinmosternte, Kapitel 8 Land- und Forstwirtschaft, Fischerei, Tabelle 8.27, Verwendete Daten: Jahre 1993-1994. In: Statistisches Bundesamt. Statistisches Jahrbuch 1995 für die Bundesrepublik Deutschland. Wiesbaden: Verlag MetzlerPoeschel, Stuttgart; 1995: S. 178, http://www.digizeitschriften.de/ $\mathrm{dms} /$ toc/?PID = PPN635628112_1995

[75] Statistisches Bundesamt. Weinmosternte, Kapitel 8 Land- und Forstwirtschaft, Fischerei, Tabelle 8.27, Verwendete Daten: Jahre 1995-1996. In: Statistisches Bundesamt. Statistisches Jahrbuch 1997 für die Bundesrepublik Deutschland. Wiesbaden: Verlag MetzlerPoeschel, Stuttgart; 1997: S. 176, http://www.digizeitschriften.de/ $\mathrm{dms} /$ toc/?PID = PPN635628112_1997

[76] Statistisches Bundesamt. Weinmosternte, Kapitel 8 Land- und Forstwirtschaft, Fischerei, Tabelle 8.24, Verwendete Daten: Jahre 1997-1998. In: Statistisches Bundesamt. Statistisches Jahrbuch 1999 für die Bundesrepublik Deutschland. Wiesbaden: Verlag MetzlerPoeschel, Stuttgart; 1999: S. 174, http://www.digizeitschriften.de/ $\mathrm{dms} /$ toc/?PID = PPN635628112_1999

[77] Statistisches Bundesamt. Weinmosternte, Kapitel 8 Land- und Forstwirtschaft, Fischerei, Tabelle 8.26, Verwendete Daten: Jahre 1999-2000. In: Statistisches Bundesamt. Statistisches Jahrbuch 2001 für die Bundesrepublik Deutschland.Wiesbaden: SFG Servicecenter Fachverlage Reutlingen; 2001: S. 176 [zugegriffen am: 20.02.2017] http://www.digizeitschriften.de/dms/ toc/?PID = PPN635628112_2001 
[78] Statistisches Bundesamt. Weinmosternte, Kapitel 8 Land- und Forstwirtschaft, Fischerei, Tabelle 8.27, Verwendete Daten: Jahre 2001-2002. In: Statistisches Bundesamt. Statistisches Jahrbuch 2003 für die Bundesrepublik Deutschland. Wiesbaden: SFG Servicecenter Fachverlage Reutlingen; 2003: S. 178 [zugegriffen am: 20.02.2017] http://www.digizeitschriften.de/dms/ toc/?PID = PPN635628112_2003

[79] Statistisches Bundesamt. Weinmosternte, Kapitel 13, Land- und Forstwirtschaft, Tabelle 13.22, Verwendete Daten: Jahre 2003-2004 In: Statistisches Bundesamt. Statistisches Jahrbuch 2005 für die Bundesrepublik Deutschland. Wiesbaden: SFG Servicecenter Fachverlage Reutlingen; 2005: S. 350 [zugegriffen am: 20.02.2017] http://www.digizeitschriften.de/dms/ toc/?PID = PPN635628112_2005

[80] Statistisches Bundesamt. Weinmosternte, Kapitel 13, Land- und Forstwirtschaft, Tabelle 13.22, Verwendete Daten: Jahre 2005-2006. In: Statistisches Bundesamt. Statistisches Jahrbuch 2007 für die Bundesrepublik Deutschland. Wiesbaden: SFG Servicecenter Fachverlage Reutlingen; 2007: S. 348

[81] Statistisches Bundesamt. Weinmosternte, Kapitel 13, Land- und Forstwirtschaft, Tabelle 13.24, Verwendete Daten: Jahre 2007-2008. In: Statistisches Bundesamt. Statistisches Jahrbuch 2009 für die Bundesrepublik Deutschland. Wiesbaden: SFG Servicecenter Fachverlage Reutlingen; 2009: S. 354

[82] Statistisches Bundesamt. Weinmosternte, Kapitel 13, Land- und Forstwirtschaft, Tabelle 13.18, Verwendete Daten: Jahre 2009-2010. In: Statistisches Bundesamt. Statistisches Jahrbuch 2011 für die Bundesrepublik Deutschland.Wiesbaden: SFG Servicecenter Fachverlage Reutlingen; 2011: S. 354 [zugegriffen am: 20.02.2017] https://www.destatis.de/DE/Publikationen/StatistischesJahrbuch/ Statistisches/ahrbuch2011.html

[83] Statistisches Bundesamt. Fachserie 3, Reihe 3.2.1 Land- und Forstwirtschaft, Fischerei. Wachstum und Ernte - Weinmost., 2013. Verwendete Daten: Jahre 2011-2012. Wiesbaden. 2014; https:// www.destatis.de/GPStatistik/receive/DEHeft_heft_00023683

[84] Statistisches Bundesamt. Fachserie 3, Reihe 3.2.1 Land- und Forstwirtschaft, Fischerei. Wachstum und Ernte - Weinmost., 2015. Verwendete Daten: Jahre 2013-2014. Wiesbaden. 2016; https:// www.destatis.de/GPStatistik/receive/DEHeft_heft_00040838

[85] Statistisches Landesamt Rheinland-Pfalz. Weinmosternte 1950 bis 2015, Verwendete Daten: Jahre 1950 und 1954. Bad Ems 2016. http://www.statistik.rlp.de/wirtschaft/landwirtschaft/tabellen/ we $1 /$ ? Fsize $=0$

[86] Uhl A, Bachmeyer S, Kobrna U et al. Handbuch Alkohol - Österreich: Zahlen, Daten, Fakten, Trends 2009. Wien: Bundesministerium für Gesundheit; 2009

[87] Kelch K, Hohmann C. Inlandsabsatz der 75 größten Biermarken. Brauwelt 2015; 17-18: 476-479

[88] Bundesministerium der Justiz und für Verbraucherschutz. Weinverordnung. Anlage 8 (zu § 17) Tabelle zur Ermittlung des natürlichen Alkoholgehalts in Volumenprozent aus dem Oechslegrad: 1995 [zugegriffen am: 14. 07. 2016]. https://www.gesetze-im-internet.de/ weinv_1995/BJNR063010995.html

[89] StataCorp LP. Stata 14.2 for Windows. 4905 Lakeway Drive, College Station, Texas 77845. USA: StataCorp LP; 2016

[90] Statistisches Bundesamt. Bevölkerung für die Jahre 1950-1969 nach Alters- und Geburtsjahren, Tabellen. Wiesbaden: Statistisches Bundesamt; 2015

[91] Statistisches Bundesamt. Fortschreibung des Bevölkerungsstandes, Code 12411-0005, Bevölkerung: Deutschland, Stichtag. Verwendete Daten: Jahre 1970 - 1989. Wiesbaden: Statistisches Bundesamt; 2016https://www-genesis.destatis.de/genesis/online
[92] Statistisches Bundesamt. Bevölkerung am Jahresende 1990-2012. Verwendete Daten: 1990 bis 2010. Wiesbaden: Statistisches Bundesamt; 2016; http://www.gbe-bund.de

[93] Statistisches Bundesamt. Bevölkerung am Jahresende ab 2011. Verwendete Daten: Jahre 2011-2014. Wiesbaden: Statistisches Bundesamt; 2016; http://www.gbe-bund.de

[94] Kim HJ, Fay MP, Feuer EJ et al. Permutation tests for joinpoint regression with applications to cancer rates. Stat Med. 2000; 19 335-351

[95] National Cancer Institute. Joinpoint regression program. Version 4.1.1.3: National Cancer Institute 2014

[96] Doll R, Peto R, Boreham J et al. Mortality in relation to smoking: 50 years‘ observations on male British doctors. BM] 2004; 328: 1519

[97] $\mathrm{Ng} \mathrm{M}$, Freeman MK, Fleming TD et al. Smoking prevalence and cigarette consumption in 187 countries, 1980-2012. JAMA 2014; 311: 183-192

[98] Orzechowski W, Walker RC. The tax burden of tobacco. Arlington, Virginia: Orzechowski and Walker; 2012

[99] Agaku IT, Alpert HR. Trends in annual sales and current use of cigarettes, cigars, roll-your-own tobacco, pipes, and smokeless tobacco among US adults, 2002-2012. Tob Control 2016; 25: 451-457

[100] California Department of Public Health. California tobacco facts and figures 2015: 2015 [zugegriffen am: 14.03. 2016]. http://www.cdph. ca.gov/programs/tobacco/Documents/Resources/ FactSheets/2015FactsFigures-web2.pdf

[101] Gual A, Colom J. Why has alcohol consumption declined in countries of southern Europe? Addiction 1997; 92: (Suppl 1): S21-S31

[102] California Department of Health Services. California Tobacco Control Update 2006. Sacramento, CA: California Department of Health Services; 2006 https://www.cdph.ca.gov/programs/tobacco/ Documents/ArchivedFiles/CTCPUpdate2006.pdf

[103] Wagenaar AC, Burris S.editors. Public health law research. San Francisco: Jossey-Bass; 2013

[104] Hanewinkel R, Isensee B. Five in a row-reactions of smokers to tobacco tax increases: population-based cross-sectional studies in Germany 2001-2006. Tob Control 2007; 16: 34-77

[105] Guindon GE, Tobin S, Yach D. Trends and affordability of cigarette prices: ample room for tax increases and related health gains. Tob Control 2002; 11: 35-43

[106] Schaller K, Braun S, Viarisio V et al. Tabakprävention in Deutschland - Was wirkt wirklich?: 2014 [zugegriffen am: 11.03.2016]. http:// www.dkfz.de/de/tabakkontrolle/download/Publikationen/AdWfP/ AdWfP_Tabakpraevention_in_Deutschland_was_wirkt_wirklich.pdf

[107] Joossens L, Raw M. The Tobacco Control Scale 2013 in Europe. Brussels: Association of European Cancer Leagues; 2014

[108] Kraftfahrtbundesamt. Bestand in den Jahren 1960 bis 2016 nach Fahrzeugklassen: 2016 [zugegriffen am: 14.07.2016]. http://www. kba.de/DE/Statistik/Fahrzeuge/Bestand/ FahrzeugklassenAufbauarten/b_fzkl_zeitreihe.html

[109] Albrecht M, Langner S. Suchtmittel im Straßenverkehr 2014 - Zahlen und Fakten. In: Suchtfragen DHf, Jahrbuch Sucht 2016. Lengerich: Pabst; 2016: S. 161-172

[110] Albrecht M, Heinrich S, Schulze H. Suchtmittel im Straßenverkehr 2008 - Zahlen und Fakten. In: Deutsche Hauptstelle für Suchtfragen, Jahrbuch Sucht 2010. Geesthacht: Neuland; 2010: S. 138-150

[111] Martineau F, Tyner E, Lorenc T et al. Population-level interventions to reduce alcohol-related harm: an overview of systematic reviews. Prev Med. 2013; 57: 278-296

[112] Räth N. Rezessionen in historischer Betrachtung. Wirtschaft und Statistik 2009; 3: 203-208 
[113] Bundesagentur für Arbeit. Zeitreihe zur Arbeitslosigkeit seit 1950 nach Strukturmerkmalen (Monats-/Jahreszahlen) - Deutschland: 2016 [zugegriffen am: 20.07.2016]. http://statistik.arbeitsagentur. de/nn_217700/Statischer-Content/Rubriken/Arbeitslose-und-gemeldetes-Stellenangebot/Arbeitslose/Arbeitslosigkeit-in-Deutschlandseit-1950-Monats-Jahreszahlen.html

[114] Mackenbach JP, Stirbu I, Roskam AJ et al. Socioeconomic inequalities in health in 22 European countries. N Engl J Med 2008; 358: 2468-2481

[115] Schaap MM, Kunst AE, Leinsalu M et al. Female ever-smoking, education, emancipation and economic development in 19 European countries. Soc Sci Med 2009; 68: 1271-1278

[116] Giskes K, Kunst AE, Benach J et al. Trends in smoking behaviour between 1985 and 2000 in nine European countries by education. J Epidemiol Community Health 2005; 59: 395-401

[117] Grittner U, Kuntsche S, Gmel G et al. Alcohol consumption and social inequality at the individual and country levels-results from an international study. Eur J Public Health 2013; 23: 332-339

[118] Probst C, Roerecke M, Behrendt S et al. Gender differences in socioeconomic inequality of alcohol-attributable mortality: A systematic review and meta-analysis. Drug Alcohol Rev 2015; 34 : 267-277

[119] Probst C, Roerecke M, Behrendt S et al. Socioeconomic differences in alcohol-attributable mortality compared with all-cause mortality: a systematic review and meta-analysis. Int J Epidemiol 2014; 43: 1314-1327
[120] Statistisches Bundesamt. Fachserie 11, Reihe S 2: Allgemeinbildende und berufliche Schulen 1950 bis 1999. Stuttgart: Metzler-Poeschel; 2000

[121] Statistisches Bundesamt. Fachserie 11, Reihe 1: Bildung und Kultur. Allgemeinbildende Schulen. Schuljahr 2013/2014. Wiesbaden: Statistisches Bundesamt; 2014

[122] John U, Hanke M. Lung cancer mortality and years of potential life lost among males and females over six decades in a country with high smoking prevalence: an observational study. BMC Cancer 2015; 15: 876

[123] Piontek D, Kraus L, Müller S et al. To what extent do age, period, and cohort patterns account for time trends and social inequalities in smoking. Sucht 2010; 56: 361

[124] Kraus L, Piontek D, Atzendorf J. Gomes de Matos E. Zeitliche Entwicklungen im Substanzkonsum in der deutschen Allgemeinbevölkerung. Sucht 2016; 62: 283-294

[125] John U, Hanke M. Liver cirrhosis mortality, alcohol consumption and tobacco consumption over a 62 year period in a high alcohol consumption country: a trend analysis. BMC Res Notes 2015; 8: 822

[126] Deutsches Weininstitut. Deutscher Wein Statistik. 2016: 2016 [zugegriffen am: 20.07.2016]. http://www.deutscheweine.de/ fileadmin/user_upload/Website/Service/Downloads/Statistik_2015-2016.pdf

[127] Bundesministerium der Justiz und für Verbraucherschutz. Schaumwein- und Zwischenerzeugnissteuergesetz: 2011 [zugegriffen am: 14.07.2016]. https://www.gesetze-im-internet.de/schaumwzwstg_2009/index.html - BJNR189600009BJNE003001000 